\title{
Importance and Benefits of Patient Registries for Duchenne Muscular Dystrophy
}

\author{
Olivia Schreiber-Katz \\ Resident Physician, Friedrich-Baur-Institute, Department of Neurology, Ludwig-Maximilians-University of Munich, Munich, Germany
}

\begin{abstract}
Rare diseases represent a special challenge for the translation of new therapies into patient care. Duchenne muscular dystrophy (DMD) is a rare childhood-onset muscular dystrophy; curative treatment is not yet available. However, new therapies are emerging to fight this devastating disease. In order to establish an infrastructure to improve data harmonisation, knowledge on the disease, public awareness, industrial interest and trial readiness, patient registries are an indispensable resource. This article provides a short overview on their importance and benefits towards improving diagnosis and care.
\end{abstract}

\section{Keywords}

Duchenne muscular dystrophy (DMD), translation, patient registries, health care burden, health economics evaluation

Disclosures: Olivia Schreiber-Katz is a member of the German Muscular Dystrophy Network (MD-NET; 01GM0887), funded by the German Ministry of Education and Research (BMBF, Bonn, Germany) from 2003 until 2013. MD-NET is a partner of TREAT-NMD (EC, 6th FP, proposal 036825; www.treat-nmd.eu). Technical setup of the German DMD patient registry was funded by the German patient organisations "aktion benni \& co" and the "Deutsche Gesellschaft für Muskelkranke e.V.", and health care evaluations were supported by the Friedrich-Baur-Stiftung, Burgkunstadt. No funding was received for the publication of this article.

Open Access: This article is published under the Creative Commons Attribution Noncommercial License, which permits any non-commercial use, distribution, adaptation and reproduction provided the original author(s) and source are given appropriate credit.

Received: 25 March 2015 Accepted: 9 April 2015 Citation: European Neurological Review, 2015;10(1):79-80 DOI: 10.17925/ENR.2015.10.01.79

Correspondence: Olivia Schreiber-Katz, Friedrich-Baur-Institute, Department of Neurology, Ludwig-Maximilians-University of Munich, Ziemssenstrasse 1, 80336 Munich, Germany. E: olivia.schreiber-katz@med.Imu.de

Duchenne muscular dystrophy (DMD) is the most common X-chromosomal inherited muscle disease in children. Nevertheless, having an estimated incidence of 1:5,000 male newborns, it is classified as a rare disorder. ${ }^{1}$ $\mathrm{DMD}$ results in progressive muscle weakness and severe disability, cardiopulmonary comorbidity and reduced life expectancy. Affected boys are limited in their independency, private and public roles, education and professional lives. No curative treatment is yet available, but symptomatic and interdisciplinary medical care represent the most important components for improving the outcome and quality of life of these patients. ${ }^{2,3}$ New curative therapies are under development, ${ }^{4}$ but rare disorders present a peculiar challenge to the translational process from bench to bedside.

\section{Prerequisites for Translation}

Translation from bench to bedside - the invention of new therapies covers the progress from preclinical research to human application and clinical trials, to market authorisation, and to implementation into standard patient care. During this process, rare neuromuscular diseases face some well-known bottlenecks: (a) lack of harmonisation, (b) lack of trial readiness, mainly resulting from (c) lack of suitable infrastructures facilitating the planning and realisation of clinical trials and (d) lack of professional, industry, political and wider public interest (as defined by patient organisations such as Association Francaise contre les myopathies [AFM], Deutsche Gesellschaft für Muskelkranke e.V. [DGM e.V.] and Eurordis).

Consequently, multinational collaboration is essential to pool resources, and suitable infrastructures must be created. Trial readiness implies standardised and internationally harmonised patients registries, aiming at recruiting a sufficient number of patients into clinical trials in a short time interval. To conduct state-of-the-art double-blind placebo-controlled trials (phase II/III), recruitment of 100-300 patients is mandatory to reach a sample size producing a reliable statistical outcome. ${ }^{5}$ For enrolment into the trial, patients need to fulfil genetic and clinical inclusion criteria. Because DMD is a rare disorder, recruitment of a large number of patients with strict inclusion criteria is often tedious and time-consuming, if not impossible endeavour. In 2003 well before the widespread implementation of DMD registries - it took Kirschner et al. ${ }^{6}$ more than 3 years to enrol 150 ambulant DMD patients in an investigator-driven, multicentre trial in Germany. For industrysponsored trials, a trial recruitment phase lasting more than 1 year is absolutely infeasible for economic reasons. So, a patient registry facilitating quick and efficient patient identification for enrolment may be a mandatory prerequisite for enabling clinical trials in DMD.

\section{How Can Patient Registries Support the Translational Process in Duchenne Muscular Dystrophy?}

Within the international network of excellence TREAT-NMD (funded by the EC 2007-2011), the implementation of internationally harmonised patient registries for DMD (and additional hereditary neuromuscular disorders) was highly supported. ${ }^{7.8}$ Meanwhile, extensive international cooperation and networking resulted in more than 50 national DMD registries, all adapted to a harmonised data content and providing pseudonomised data for a global database. ${ }^{9}$ Numerous clinical trials were by now facilitated by these registries. Additionally, these harmonised longitudinal data of several thousand DMD patients 
worldwide led to a global gain of knowledge regarding epidemiology, natural history, genotype-phenotype correlations and care of DMD patients all over the world. ${ }^{10,11}$

Moreover, the DMD patient registries successfully increased the awareness and visibility of this devastating disease among the wider public and created a unique possibility for industrial partners and sponsors to contact the patient community. Thus it became possible to get a clear picture of the target market, easing the feasibility and planning of clinical trials along with patient recruitment, resulting in higher investments into this rare disease. The authorisation of a new therapeutic drug to treat DMD in $2014^{12}$ is a perfect and successful example of close cooperation between sponsors and the global DMD patient registry's leading to a direct improvement of patient treatment and care.

By now, multiple national DMD registries, along with the global registry, have evolved into indispensable infrastructures advancing diagnosis and therapy in DMD having a direct effect on individual patients. Accordingly, international care guidelines have been defined and disseminated to patients and caregivers through patient registries. Additionally, registered patients benefit from feedback on new research developments as well as from a direct link to the scientific community, resulting in a feeling of 'not being left behind' and belonging to a broader network.13

\section{The Role of Patient Registries in Health Economics Evaluations}

Comprehensive socioeconomic evaluations of rare diseases are urgently needed to enable the facilitation of new therapeutic developments and current mandatory cost-benefit analysis. The clinical development of new promising therapies is costly, and after being approved by regulatory authorities, implementation in DMD care may produce high expenditures for the health care systems of individual countries. However, despite their cost, innovative therapies could also reduce the health care burden of this progressive and fatal disorder. Health economics evaluations represent the first analytical step towards a systematic health economic analysis of upcoming innovative DMD therapies, including comparative effectiveness research aiming at enabling a smooth translation of innovations from clinical research over payer negotiations and marketing authorisation to a reimbursed therapy.

Only a few studies had been published in this field before patient registries were established, with retrospective data acquisition of homogenous patient cohorts being difficult. ${ }^{14,15}$ Patient registries help define patient cohorts according to specified criteria and address patients directly, so retrospective or even prospective health economics evaluations, health services research and patients' quality-of-life evaluations can be achieved more easily.16,17 These advancements in DMD care, accomplished via the patient registries, can represent a model for other rare disorders and may contribute to a more comprehensive understanding of health care delivery matters in rare disorders in general.

\section{Conclusion}

Patient registries are a fundamental resource supporting the translational process from bench to bedside in rare disorders. In DMD, international collaboration has led to the synchronised setup of national registries and data harmonisation within a global database that helped to further characterise this rare disease, empower trial readiness and create a sustainable expert network serving as a contact platform for professionals, researchers, industrial partners, politics and patients. Patients are not left behind but are actively involved in this expert community. The most important previous achievements brought about with the help of the registries were the definition and dissemination of standards of care, the authorisation of a new therapeutic drug for DMD and the implementation of health care evaluations in DMD.
1. Mendell JR, Lloyd-Puryear M, Report of MDA muscle disease symposium on newborn screening for Duchenne muscular dystrophy, Muscle Nerve, 2013;48:21-6.

2. Bushby K, Finkel R, Birnkrant DJ, et al., Diagnosis and management of Duchenne muscular dystrophy, part 1: diagnosis, and pharmacological and psychosocial 1. diagnosis, and pharmacological and psych
management, Lancet Neurol, 2010;9:77-93.

3. Bushby $K$, Finkel R, Birnkrant DJ, et al., Diagnosis and management of Duchenne muscular dystrophy, part 2: management of Duchenne muscular dystrophy, part 2:
implementation of multidisciplinary care, Lancet Neurol implementation of

4. Aartsma-Rus A, Van Ommen GJ, Kaplan JC, Innovating therapies for muscle diseases, Handb Clin Neurol, 2013;113:1497-501.

5. Aartsma-Rus A, The risks of therapeutic misconception and individual patient $(\mathrm{n}=1)$ "trials" in rare diseases such as Duchenne dystrophy, Neuromuscular Disord, 2011;21:13-5.

6. Kirschner J, SchessI J, Schara U, et al., Treatment of Duchenne muscular dystrophy with Ciclosporin A: a randomised, double-blind, placebo-controlled multicentre trial, Lancet Neurol, 2010;9:1053-9.
7. Bushby K, Lynn S, Straub T, et al., Collaborating to bring new therapies to the patient-the TREAT-NMD model, Acta Myol, 2009;28:12-5.

8. Sarkozy A, Bushby K, Beroud C, et al., 157th ENMC International Workshop: patient registries for rare, inherited muscular disorders 25-27 January 2008, Naarden, The Netherlands, Neuromuscular Disord, 2008:18:997-1001.

9. Bladen CL, Rafferty K, Straub V, et al., The TREAT-NMD Duchenne muscular dystrophy registries: conception, design, Duchenne muscular dystrophy registries: conception, design, and utilization

10. Bladen CL, Salgado D, Monges S, et al., The TREAT-NMD DMD Global Database: analysis of more than 7,000 Duchenne muscular dystrophy mutations, Hum Mutat, 2015;36:395-402

11. Rodger $\mathrm{S}$, Woods $\mathrm{KL}$, Bladen $\mathrm{CL}$, et al., Adult care for Duchenne muscular dystrophy in the UK, J Neurol, 2015;262:629-41

12. Haas M, Vlcek V, Balabanov P, et al., European Medicines Agency review of ataluren for the treatment of ambulant patients aged 5 years and older with Duchenne muscular dystrophy resulting from a nonsense mutation in the dystrophin gene, Neuromuscular Disord, 2015;25:5-13.

13. The diagnosis and management of Duchenne muscular dystrophy: a guide for families, 2010. Available at: www.treatnmd.eu/downloads/file/standardsofcare/dmd/us_english/ dmd_us_familyguide_june2010.pdf (accessed 9 May 2015).

14. Ouyang L, Grosse SD, Kenneson A, et al., Health care utilization and expenditures for children and young adults with muscular dystrophy in a privately insured population, J Child Neurol, 2008;23:883-8.

15. Larkindale J, Yang W, Hogan PF, et al., Cost of illness for neuromuscular diseases in the United States, Muscle Nerve, 2014;49:431-8

16. Landfeldt $E$, Lindgren $P$, Bell $C F$, et al., The burden of Duchenne muscular dystrophy: An international, crosssectional study, Neurology, 2014;83:529-36.

17. Schreiber-Katz $\mathrm{O}$, Klug $\mathrm{C}$, Thiele $\mathrm{S}$, et al., Comparative cost of illness analysis and assessment of health care burden of Duchenne and Becker muscular dystrophies in Germany, Orphanet J Rare Dis, 2014:9:210. 